\title{
PERSPECTIVES
}

OPINION

\section{Is HIV- 1 evolving to a less virulent form in humans?}

Kevin K. Ariën, Guido Vanham and Eric J. Arts

Abstract | During the rapid spread of HIV-1 in humans, the main (M) group of HIV-1 has evolved into ten distinct subtypes, undergone countless recombination events and diversified extensively. The impact of this extreme genetic diversity on the phenotype of HIV-1 has only recently become a research focus, but early findings indicate that the dominance of HIV-1 subtype $C$ in the current epidemic might be related to the lower virulence of this subtype compared with other subtypes. Here, we explore whether HIV-1 has reached peak virulence or has already started the slow path to attenuation.

There are four classified retroviruses that infect humans and cause symptomatic disease. Human T-cell lymphotropic virus (HTLV) types 1 and 2 are members of the Deltaretrovirus genus in the subfamily Orthoretrovirinae, show a low rate of evolution in the human population, and probably originated from a non-human primate millions of years ago ${ }^{1-4}$. The high frequency of HTLV-1 infections was localized in specific endemic areas (for example, southern Japan) until recent times, indicating a relatively slow spread in the human population compared with another human retrovirus, HIV-1.

HIV-1 was introduced into the human population just $60-80$ years ago ${ }^{5}$ but an estimated 40 million individuals are currently infected with the virus ${ }^{6}$. The spread and expansion of HIV-1 across Africa and throughout the world has been accompanied by one of the most rapid evolutionary rates described for a human pathogen ${ }^{6}$, aside from hepatitis $\mathrm{C}$ virus. HIV-1 remains one of the most lethal pathogens ( $100 \%$ mortality) that currently infects humans, whereas infection by other human viruses that are often more feared, such as Ebola, severe acute respiratory syndrome (SARS), influenza H5NI and Lassa Fever, can have a mortality rate of $<50 \%$ (REFS 7-9).

Whether or not a lethal pathogen evolves to become a relatively benign parasite or even a commensal organism has been a subject of great debate. The survival of Euroasian populations during the European bubonic plague (1347-1352) and the 1918 influenza epidemic is often perceived as evidence for pathogen attenuation. However, neither Yersinia pestis nor the H1N1 influenza virus seem to have attenuated during these relatively short human epidemics. Surviving the $Y$. pestis epidemic is thought to be more related to fractionation of the human population, the resistance of rodent ectoparasites and, possibly, the selection of resistant human hosts ${ }^{10,11}$.

Nonetheless, there are many examples of microparasite evolution and host selection leading to attenuation or resistance to disease. The best documented and controlled example is the rapid outgrowth of an attenuated myxoma virus following its introduction into Australia in 1950 to control the expanding rabbit population ${ }^{12-14}$. This virus might have attenuated as a result of altered immunomodulatory properties in addition to a reduction in the replication rate $^{13,14}$. More recent analyses of archived reports from the fifteenth century suggest that the dramatic decrease in the severity of syphilis symptoms that took place over a period of less than 10 years was related to a reduction in virulence rather than the selection of resistant hosts ${ }^{15}$. Rapid (decades to centuries) versus slow (thousand to millions of years) attenuation might be related to the severity of disease, the rate of lethality and the transmission efficiency or transmission routes. The suggestion that the simian lentiviruses have attenuated in their non-human primate hosts during millions of years of co-evolution ${ }^{16}$ has been challenged by recent phylogenetic analyses that indicate the introduction of simian immunodeficiency virus (SIV) into African primates might be a relatively recent event that unfolded in the past hundreds or thousands of years, therefore indicating that primate lentiviruses might frequently jump between primate species and then rapidly adapt to the new host $\mathrm{t}^{17,18}$.

Studies that have modelled the expansion and contraction of various epidemics have indicated that the inter-relationships between lethal pathogens and their hosts are complex and frequently not comparable ${ }^{19-22}$. These questions can only be answered by understanding pathogen evolution, dynamics and spread within the population at large and within infected hosts. The propensity for HIV-1 to evolve rapidly in response to various immune and other host pressures indicates that this virus is more likely to become attenuated through a reduction of virulence rather than selection of resistant hosts.

Defining pathogen fitness and virulence The phylogenetic approaches that are commonly used to describe the evolution of pathogens do not examine how these genetic differences actually impact on the physical attributes, often termed the fitness, of the pathogen. This is understandable, given the complexities of measuring fitness, which can be defined as the adaptability or reproductive success of an organism in a specific environment. In the case of HIV-1 research, fitness is frequently misrepresented or overinterpreted $^{23,24}$. For example, HIV-1 virions in which the reverse transcriptase (RT) harbours mutations that confer resistance to antiretroviral drugs (ARVs) such as 3TC (Lamivudine or Epivir) seem less fit than virions carrying the wild-type RT ${ }^{25,26}$. However, this reduced fitness in a reconstituted in vitro assay does not necessarily imply that the drug-resistant virus will be less fit in a human host receiving ARVs. Fitness in each 
environment can be inter-related but is not necessarily consequential. For the purposes of this Opinion article, HIV-1 fitness will be defined in a specific environment and only significant correlations between environments will be highlighted as being potentially consequential.

Fitness is not always synonymous with virulence, which is typically defined as the rate of host mortality as a consequence of infection ${ }^{27}$ but which can be further refined to include the reproduction rate and pathogenic potential of the pathogen ${ }^{28}$. These parameters also define the fitness of a pathogen within a host. Typically, virulence is difficult to measure for viruses such as HIV-1, given the long asymptomatic period after infection. Therefore, intermittent measures of HIV-1 replicative fitness during disease can often provide insights into the rates of disease progression ${ }^{29-32}$. Confusion between these principles of virulence and fitness is introduced when examining fitness within the host compared with examining fitness within a population ${ }^{24,33}$. Maynard, Ewald, Anderson, May, Novak and others $^{22,33-36}$ suggest that pathogen strain 'A', with slow replicative fitness but high transmission efficiency, will be favoured in a host population over strain ' $B$ ', with increased replicative fitness. However, when the strains compete together in a single host, it is clear that strain ' $\mathrm{B}$ ' is more virulent and would out-compete and, consequently, be fitter than, strain ' $\mathrm{A}$ '. In terms of a pathogen spreading through a host population, high pathogen fitness is then defined by a reduction in reproduction rate and virulence without a loss in transmission. This article will address the possible direct relationship between HIV-1 virulence and replicative fitness but it is important to note that attenuation of virulence is not always synonymous with lower replicative capacity. For example, the attenuation of myxoma virulence in the Australian rabbit population might be more related to changes in virus-mediated immunomodulation than to lower replicative fitness in rabbits ${ }^{13,14}$.

\section{Expansion of HIV-1 in humans}

HIV-1 was first introduced into the human population from the chimpanzee subspecies Pan troglodytes troglodytes ${ }^{37-39}$. Most recently, Keele et al. have discovered a region in southern Cameroon where chimpanzees carry SIV strains $\left(\mathrm{SIV}_{\text {cpzptt }}\right)$ that are closely related to two distinct lineages of HIV-1, the main (M) and new (N) groups ${ }^{37}$ (FIG. 1). The origin of the outlier $(\mathrm{O})$ group might be related to a jump from Gorilla gorilla ${ }^{40}$. SIV cpzptt $_{\text {was intro- }}$ duced into the human population multiple times, over decades to possibly centuries, but these transfer events probably started to increase in the 1920s to the 1950s as a result of human migration into this dense tropical region ${ }^{5,37-39}$. Similar to the pending introduction of influenza $\mathrm{H} 5 \mathrm{~N} 1$ from the avian to the human host ${ }^{41}$, a distinct but possibly rare SIV $_{\text {cpzptt }}$ strain might have had the capacity for efficient replication and sexual transmission in humans $s^{17,37}$. Following this founder event, human population density and contact had to be sufficiently high for subsequent transmission and spread. It is possible that a single transmission of HIV-1 group $M$ spawned the $>60-80$ million infections that have taken place since the beginning of the epidemic $^{37}$. By contrast, HIV-2 originated in humans from a cross-species transmission from sooty mangabeys in west Africa (near or in Guinea-Bissau) around 1930-1955 (REFS 42,43). In the 1980s, the incidence of HIV-1 group M in central Africa and HIV-2 in west Africa expanded exponentially but clearly at different rates, as the prevalence of HIV-1 M infections (28 million) far exceeds that of HIV-2 infections ( $<1$ million $)^{6,44,45}$. In stark contrast, $\mathrm{HIV}-1$ groups $\mathrm{O}$ and $\mathrm{N}$ are responsible for $<25,000$ infections, most of which are in Cameroon and Gabon ${ }^{6,46-49}$. The expansion and divergent evolution of HIV-1 group M into diverse subtypes (FIG. 1) has been dated to 1956-1976 and might have coincided with human emigration and the seeding of new regional epidemics in central Africa $^{5,50,51}$.

HIV-1 subtypes share 70-90\% sequence identity, groups share $<70 \%$, and HIV-1 and HIV-2 can differ as much as $50 \%$ at the nucleotide level (FIG. 1). Given this extreme genetic diversity, is it reasonable to assume that not all HIV types, groups, subtypes and even isolates have evolved to maintain similar virulence? Although many research articles have described genotypic differences between subtypes, there are only a handful of studies that have examined potential phenotypic differences among the human lentiviruses ${ }^{52-56}$. No studies have modelled how phenotypic or 'fitness' differences among these viruses might affect disease progression in infected individuals or the general spread of the virus in the human population. This article can only provide predictions of temporal HIV-1 attenuation based on published studies and significant trends during the human epidemic. To understand how attenuation of virulence can occur, it is important to understand the population dynamics and fitness of HIV-1 in each infected host and during host-to-host transmission.
How HIV-1 infects, and evolves in, a host In relation to other sexually transmitted pathogens, HIV-1 is transmitted with moderate efficiency by sexual contact and apparently greater efficiency by direct blood-to-blood contact (for example, needle sharing by intravenous drug users) ${ }^{57,58}$ or vertical mother-to-child perinatal transmission $^{59}$. There are many cases of discordant couples in which the HIV-positive individual fails to transmit the virus to the uninfected partner despite frequent opportunity ${ }^{60}$. A schematic of transmission events and disease progression is provided in FIG. 2 and BOX 1. An increased viral load in the donor and consequently in the inoculating dose increases the transmission efficiency ${ }^{61,62}$. An increased viral load in the donor also corresponds to greater genetic diversity of the inoculating HIV-1 population (termed the virus isolate) ${ }^{63}$ (FIG. 2), and yet few HIV-1 clones will establish an infection in the recipient ${ }^{64}$. This dramatic bottleneck might be related to accessing the initial target cells (cells of the dendritic lineage $)^{65,66}$ and establishing a productive, systemic infection. Based on extensive studies on RNA viruses, it is clear that restrictive genetic bottlenecks invoked by various selection pressures will reduce replicative fitness ${ }^{67-69}$. Unfortunately, nearly all of these viral fitness studies have been done in tissue-culture infections and not in animal models or in defined donor-recipient transmission pairs. Greater HIV-1 genetic diversity during acute or early infection has been associated with more rapid disease progression $^{70}$. In addition, the genetic diversity of HIV-1 seems to correlate directly with replicative fitness ${ }^{29}$.

HIV is the obvious aetiological agent of AIDS, yet it is commonly assumed that the phenotypic characteristics and replication efficiency (the ex vivo fitness) of the infecting, wild-type HIV isolates have little impact on the rate of disease progression. Much attention has been focused on host correlates of disease progression, such as the strength of HIV-specific immune responses ${ }^{71-77}$ and host genetic polymorphisms that might affect HIV1 replication ${ }^{78-82}$. RNA load remains the best predictor of HIV disease progression ${ }^{83}$ and the dramatic reductions in this load achieved by ARV treatment can delay disease progres$\operatorname{sion}^{84}$. It is important to note that a high viral load is not an absolute in relation to the severity of the disease. In non-human hosts such as sooty mangabeys, a high SIV load is not associated with clinical symptoms ${ }^{85}$. Recent studies indicate that the decline in $\mathrm{CD} 4{ }^{+} \mathrm{T}$-cell counts, and not the increase in viral load, is a better predictor of disease progression ${ }^{86}$. 


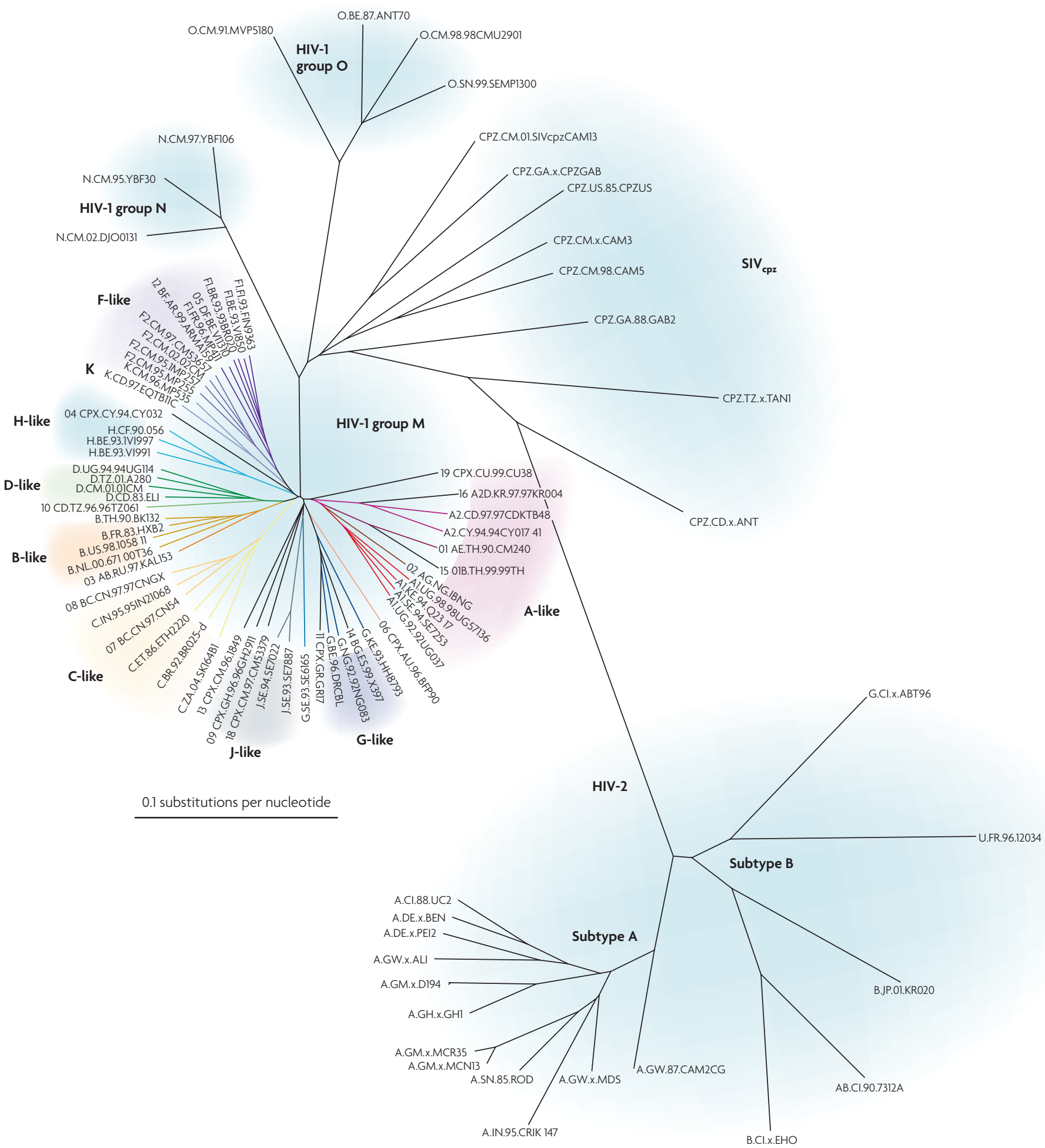

Figure 1 | Phylogenetic tree of human and simian lentiviruses. The genetic similarity between different HIV and simian immunodeficiency virus (SIV) strains was compared by aligning the full genome sequences of 87 human and simian lentiviruses using ClustalX v.1.83 (the accession numbers are available on request). Phylogenetic trees based on nucleotide distance were constructed by neighbour-joining methods as implemented in ClustalX with 1,000 bootstrap resamplings (not presented) and schematically represented with the TreeView program. HIV-2 and HIV-1 share only $50-60 \%$ sequence identity and cluster at distinct locations on the phylogenetic tree whereas SIV $_{\mathrm{cpz}}$ branches out from the root of the HIV-1 groups. The origins of these HIV-1 groups in southern Cameroon have recently been described and indicate two probable jumps from chimpanzee (groups $\mathrm{M}$ and $\mathrm{N}$ ) and gorilla (group O) species. HIV-1 M subtypes probably evolved from a discrete introduction into the human population and then diverged into different subtypes. The subtypes defined as 'A-like' describe HIV-1 isolates with sequences that map phylogenetically more to subtype A than to any other subtype. For example, the recombinant form CRF02_AG (such as 02 AG.NG.IBNG in the HIV-1 group M A-like cluster) has longer genomic segments that are more related to subtype $A$ than to subtype G. M, main; N, new. 


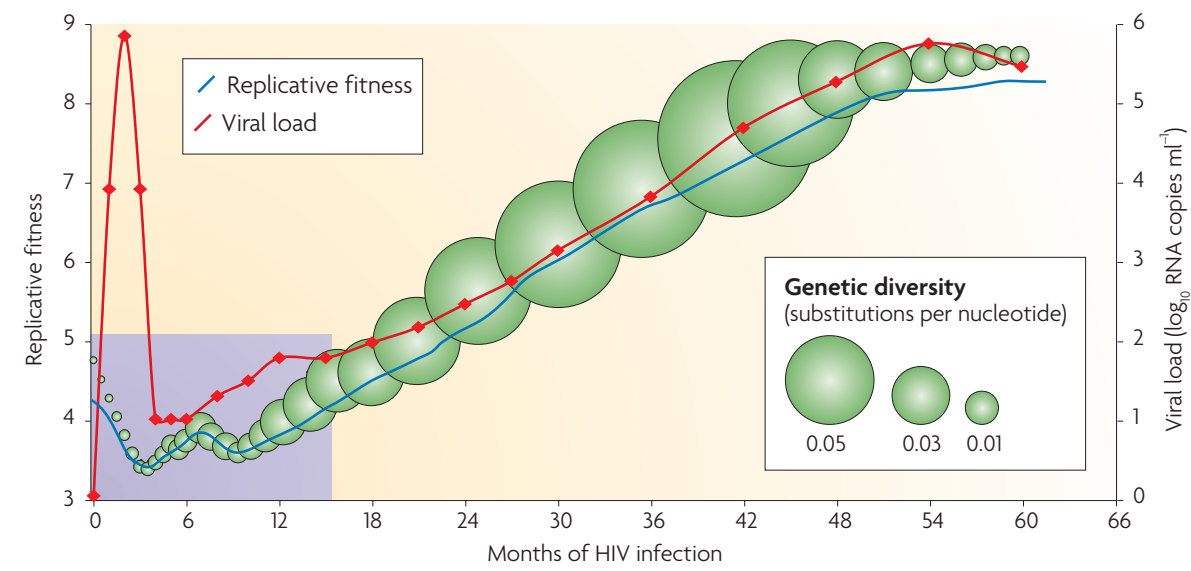

Figure 2 | A hypothetical example of changes in replicative fitness and viral load during HIV-1 disease progression. An individual is typically infected by a few HIV-1 clones (depicted by a small green circle), which dramatically increase in copy number but not in genetic diversity during the first 1-2 months of infection. Following this acute infection period, viral load is reduced partly as a result of strong HIV-specific cell-mediated immunity. The virus population is thought to oscillate between expansion of HIV-1 populations owing to immune escape from existing HIV-specific cytotoxic T-lymphocyte (CTL) clones and contraction caused by new genetic bottlenecks that are induced by newly emerging CTL clones. The replicative fitness and genetic diversity of the HIV-1 population seem to track closely together and, following early disease (purple box), both increase at a relatively linear rate with the length of infection. This increase in replicative fitness correlates with increases in viral loads (right axis) and decreases in $\mathrm{CD} 4^{+} \mathrm{T}$-cell counts (not shown). The scale for replicative fitness is arbitrary but is derived from the relative HIV-1 fitness values, that is, the ability of one HIV-1 isolate to out-compete another in ex vivo dual-virus competition experiments.

The first evidence that specific viral genetic traits might affect virulence was the identification of long-term non-progressing individuals harbouring a defective HIV-1 isolate lacking a $n e f$ open reading frame $\mathrm{e}^{87,88}$. Mutations or deletions in this accessoryprotein-coding gene can significantly impair fitness in tissue-culture infections ${ }^{89}$. Even with this discovery 10 years ago, the effect of relative HIV-1 replicative fitness on disease progression has not been the subject of intense study owing to difficulties in accurately measuring HIV-1 fitness.

There have been only limited studies examining how virus fitness changes during natural infection $s^{31,32,90,91}$. The replication capacities of different HIV-1 isolates are commonly compared by measuring ex vivo growth kinetics in single virus infections $^{31,32}$. The viruses used in many studies are not isolated from infected patients but rather are chimeric recombinant viruses containing gene(s) from the patient's virus within the genome of a laboratory HIV-1 $\operatorname{strain}^{32,92}$. Small environmental differences in separate single virus infections and the inherent variability of detection assays can prevent accurate estimates of viral fitness by these methods. By contrast, dual virus infections or competition assays involve multiple rounds of virus replication, are less affected by environmental changes and, as a result, can quantify small but significant differences in fitness ${ }^{29,93,94}$. In HIV research, multiple and single-cycle single virus infections, and competition experiments, have been used in studies that described impaired HIV-1 replicative fitness conferred by drug-resistance mutations ${ }^{95,96}$. Nevertheless, the impact of these drug resistance mutations in primary HIV-1 isolates (as opposed to chimeric viruses) has not been fully assessed.

Initial reports using HIV-1 competition assays in peripheral blood mononuclear cell (PBMC) cultures to measure fitness indicated that many long-term survivors harbour HIV-1 with an impaired replication capacity, whereas rapid progressors can be infected with more aggressive HIV-1 isolate ${ }^{30}$. Subsequent studies indicated that the genetic diversity and fitness of the intra-patient HIV-1 population continues to increase during disease and to diverge from the founder HIV-1 clones ${ }^{29,63,70}$ (FIG. 2). This diversity reaches an inflection point just before the inception of AIDS symptoms. During the asymptomatic period, the steady increase in HIV-1 diversity occurs concomitantly with increasing viral load and, more significantly, with increasing ex vivo replicative fitness of the infecting HIV-1 isolate ${ }^{29}$ (FIG. 2). Red Queen dynamics (in which the interaction between a parasite and its host leads to a constant evolutionary process of adaptation and counter-adaptation) predict that increasing replicative fitness must be associated with a continual expansion of the genetic breadth and size of a population ${ }^{97,98}$. Any strong selective pressure can result in the contraction of population size, the appearance of deleterious mutations and decreasing fitness ${ }^{99-101}$. Interestingly, the introduction of ARVs as a strong selective pressure during asymptomatic disease resulted in a dramatic decrease in HIV-1 load, genetic diversity and replicative fitness, even in the absence of drug resistance mutation ${ }^{29}$.

Following transmission, it is possible that the replicative fitness and genetic diversity of newly infecting HIV-1 isolates is lower than that of the inoculating virus from the donor (BOX 1). Such a continual increase in HIV-1 diversity and fitness is in direct conflict with the idea that the HIV-specific acquired immune response can counter this viral expansion ${ }^{102}$. Discrepancies in these data might simply be due to the time period of sampling and analyses. HIV-1 genetic diversity and fitness only increases after the first one to two years of infection ${ }^{29}$ (FIG. 2). Immediately following acute infection, both host genetics and the HIV-specific immune response might have crucial roles in establishing a genetic diversity and fitness set point, which might also correspond with the well-documented viral-load set point ${ }^{83,103}$ (FIG. 2). Cell-mediated immunity could be the most dominant factor in specifically reducing viral load and, as a consequence, fitness and diversity ${ }^{104-107}$.

\section{How can HIV-1 virulence be attenuated?}

The genes at the human leukocyte antigen (HLA) loci are extremely variable. In humans in most geographical regions, it is typically rare to find a perfect match in the HLA A-G alleles that encode major histocompatibility complex (MHC) class I and in the six HLA D genes that encode MHC class II. Even in regions of specific ethnicities, there is considerable population diversity in HLA alleles, which encompass at least 21 major $\mathrm{A}, 35 \mathrm{~B}$ and $15 \mathrm{C}$ alleles (see the dbMHC web site). Compared with Eurasian populations, HLA diversity is much greater in the main ethnic groups of sub-Saharan Africa; a study of $>4,000$ individuals characterized at the $A, B$ and $C$ loci found no preference for a single allele ${ }^{108}$. The least HLA diversity is often observed in more homogeneous Caucasian populations, most notably, the Caucasian Australian population (see the dbMHC web site) ${ }^{109}$.

How might a human population that is diverse in terms of HLA type or other host polymorphisms influence HIV-1 evolution? 
Quantitative aspects of the HIV-specific immune response (for example, high levels of HIV-specific cytotoxic T lymphocytes (CTLs) versus low levels) are self-limiting because this immunity is not transferred between individuals. However, a qualitative difference in terms of what the immune system 'sees' in the infecting HIV-1 strain could have a significant impact on virulence. A mismatch of HLA genes between the donor and recipient of a transmission pair would result in the presentation of a different set of HIV-1 peptide epitopes to $\mathrm{CD}^{+} \mathrm{T}$ cells in the recipient that were not recognized in the donor ${ }^{110,111}$. With each new transmission, the infecting virus must then evolve to escape this pressure, which in turn seems to reduce replicative fitness ${ }^{12,113}$. In human populations with a low HLA diversity (for example, in Australia), CTL escape mutations might become fixed along with compensatory mutations that restore or maintain HIV-1 virulence ${ }^{109}$ (BOX 1). By contrast, the constant passage of HIV-1 through humans of different HLA types (for example, in Africa) might prevent the accumulation of compensatory mutations, which could stabilize CTL escape mutations (BOX 1). However, a model involving only HLA types is obviously too simplistic, considering the effects of other host factors. For example, polymorphisms in other host restriction factors, such as ABOBEC $3 \mathrm{G} / 3 \mathrm{~F}$ and TRIM $5 \alpha$, could also alter disease progression $^{114,115}$. Furthermore, host polymorphisms in the HIV-1 co-receptor CCR5 and its ligands (for example, CCL3L1) alter co-receptor or ligand expression levels and, as such, affect the ability of HIV-1 to use the co-receptor ${ }^{78-82}$. If these polymorphisms exist at high frequencies in the human population, they too could result in an oscillation between HIV-1 sequences that, in this example, reduce or increase co-receptor avidity, a correlate of replicative fitness.

In general, HIV-1 virulence might attenuate if, on average, the loss in fitness that occurs following transmission is greater than the gain in replicative fitness that occurs during asymptomatic disease progression (BOX 1). Loss of fitness is probably due to the genetic bottleneck during transmission and/or escape from host restrictions or the host immune response during early disease. The continual introduction of new selective pressures and genetic bottlenecks would lead to the accumulation of deleterious mutations, which in turn would reduce replicative fitness or virulence. However, these hypotheses have not been empirically tested using HIV-1 transmission pairs.

\section{Box 1 | A model for HIV-1 attenuation}

The figure shows a model for HIV-1 attenuation in a human population with diverse and limited genetic polymorphisms that are associated with HIV-1 disease progression. In this illustration, patient $A$ is the founder who will infect patient $B$ approximately 4 years into the infection of $A$. Before transmission from patient $A$ to patient $B$, the replicative (or pathogenic) fitness of the virus has increased from 4 to 7 (arbitrary units, see FIG. 2 legend). Although the replicative fitness increases in patient $A$, the transmission from patient $A$ to patient $B$ results in a genetic bottleneck that resets the fitness to a lower baseline or set level (dotted line). As patients $A$ and $B$ are not an HLA match, the cell-mediated immune response (or CTL response) of patient $B$ recognizes a different set of HIV-1 epitopes than in patient A. In patient B, mutations in this new set of epitopes are necessary for CTL escape and for the virus to expand in the face of this anti-viral response. However, this 'escape' from the CTL response comes at a fitness cost and reduces the replicative fitness in the first 1-2 years of infection. Following this escape, the infecting HIV-1 isolates seem to continually increase in number (that is, the viral load increases) ${ }^{83}$ and gain replicative fitness ${ }^{29}$ (FIG. 2). This process is repeated with each transmission event in patients with diverse HLA alleles. Patient $B$ transmits to patient $C$ and patient $C$ transmits to patient $D$ during acute infection. Increased transmission efficiency is associated with higher viral loads, which are highest during acute infection. However, the short time interval of acute infection provides limited opportunity for transmission. As the disease progresses and viral load increases, the efficiency of transmission will increase even though opportunities for transmission can remain constant. Consequently, more transmission events might result from prolonged, compared with rapid, disease progression (for example, transmission from patient $D$ to $E$ ) even when accounting for the high transmission efficiencies during acute infection ${ }^{149,150}$. In this model, owing to the fitness bottlenecks at transmission and the subsequent loss in fitness caused by CTL escape ${ }^{111-113}$, the circulating HIV-1 isolate will start to lose virulence. This loss in virulence would decrease pathogenesis, prolong the time to AIDS or even result in a non-symptomatic infection. The complete clearance of HIV-1 is unlikely because of the stable integration of the provirus into memory T cells with long half lives and possible re-activation of virus replication in these cells ${ }^{160,161}$.

In patients with limited genetic diversity and possible HLA matches, loss of fitness might be associated with the genetic bottleneck following transmission but the infecting HIV-1 isolate could be more 'resistant' to the breadth of possible CTL responses. For example, patient A transmits HIV-1 to patient $X 1.5$ years into the infection. After 1.5 years, the HIV-1 isolate in patient A has mutated the dominant HIV-1 epitopes and escaped CTL activity. When patient $A$ infects patient $X$ (who is a match at some or all of the HLA alleles), the virus from patient A cannot be efficiently recognized by the $C T L$ response of patient $X$ and so could result in a more rapid disease progression. An HIV-1 isolate passing through patients of limited HLA diversity might actually increase in virulence. CTL, cytotoxic T lymphocyte; HLA, human leukocyte antigen.

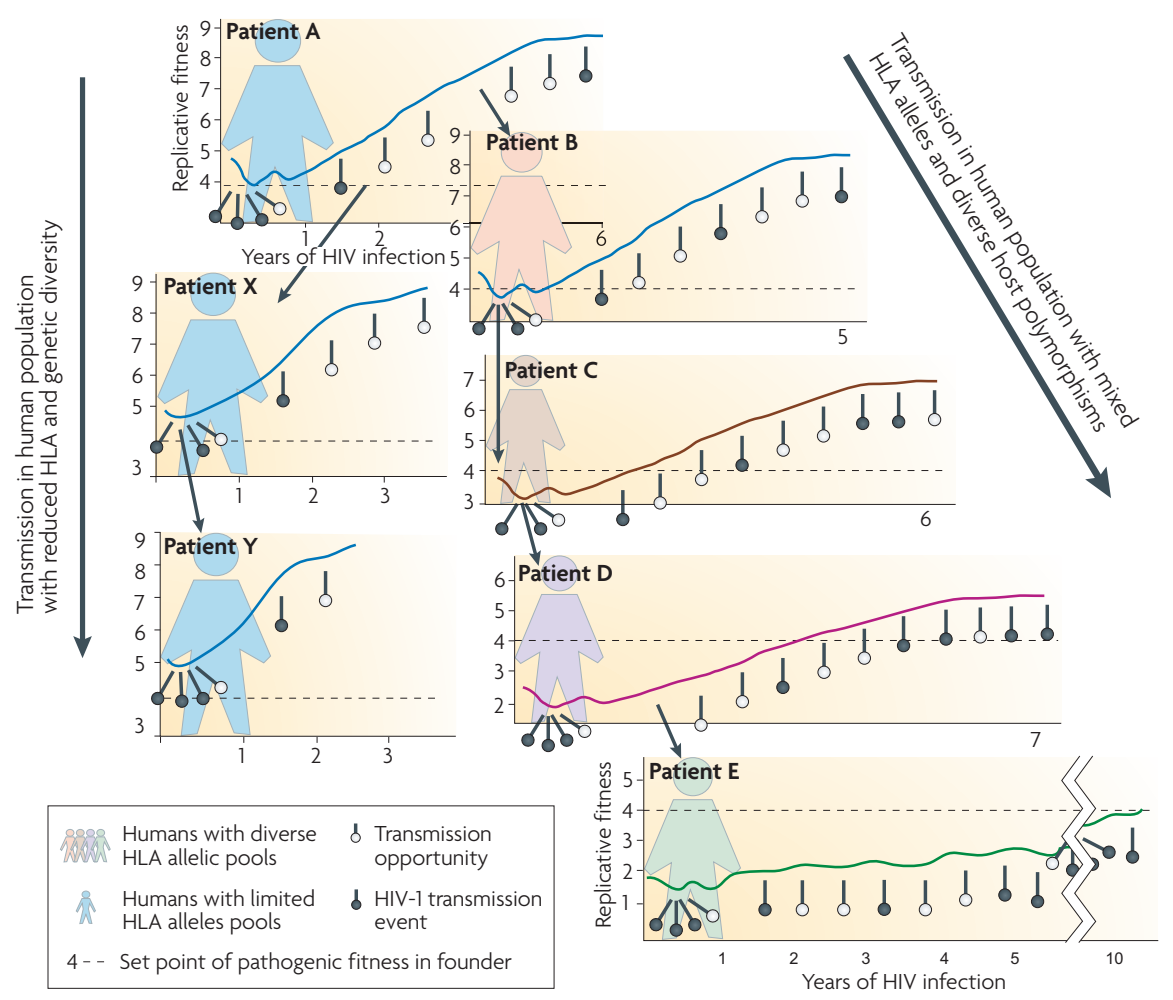


Is there evidence for attenuation of HIV-1?

With the exceptions of anecdotes, a few case reports and one published study on a large cohort, there is no evidence that $\mathrm{HIV}-1$ virulence has either increased or decreased during the past 20-30 years of the pandemic ${ }^{116-121}$. One of the main obstacles in comparing disease progression in the mid-1980s with the current day epidemic in the developed world is the significant medical advancements that have been made. In the developing world, until recently, few if any cohorts have been analysed for disease progression. Finally, owing to increased awareness and education about AIDS, patients are now diagnosed earlier, sometimes during acute infection, whereas in the 1980s many patients did not present to a clinic until the onset of AIDS. As a result, there is often the impression that HIV-1 infections are less aggressive now than they were at the beginning of the pandemic.
In a study published last year, $>100$ primary HIV-1 isolates were obtained from patient samples in Antwerp, Belgium, but only twelve could be matched from the periods 1985-1987 and 2002-2003 (REF. 121). All 24 viruses from both time periods were competed against each other in PBMC from HIV-negative donors. Based on $>500$ headto-head virus competition experiments, the recent $\mathrm{HIV}-1$ isolates were significantly less fit than the historical isolates ${ }^{121}$. These findings indicate that HIV-1 might have attenuated in replicative fitness over the past 15-18 years. If the fitness of the infecting HIV-1 strain is a predictor of disease progression $^{29-32}$, reduced ex vivo fitness in PBMC might be a strong correlate of decreased virulence. However, these results should be interpreted with caution owing to the limited sample size involved. Furthermore, the cross-sectional design of this study results in sampling of HIV-1 isolates at a single time point during disease progression, which was controlled for disease stage based on $\mathrm{CD} 4^{+}$ T-cell count and viral load. Nonetheless, the increased fitness of the 1980 viruses versus the 2000 viruses was significant and greater than any increase in HIV-1 fitness observed during disease progression ${ }^{29,121}$. Future studies on large cohorts that span the epidemic temporally in various geographical regions are warranted but, as described below, these studies will again be limited to analyses of HIV-1 virulence (that is, replicative fitness) as rates of disease progression will not be available (see below).

Rates of disease progression have now been examined in a European HIV-infected cohort spanning the years 1986-2002 (REF. 119). The slopes of CD4 ${ }^{+} \mathrm{T}$-cell decline were calculated in antiretroviral-treatmentnaive patients with confirmed dates of infection, at no fewer than nine time points over periods $>1$ year. Following this early period of the AIDS epidemic, there seems to be some evidence for slowing rates of disease

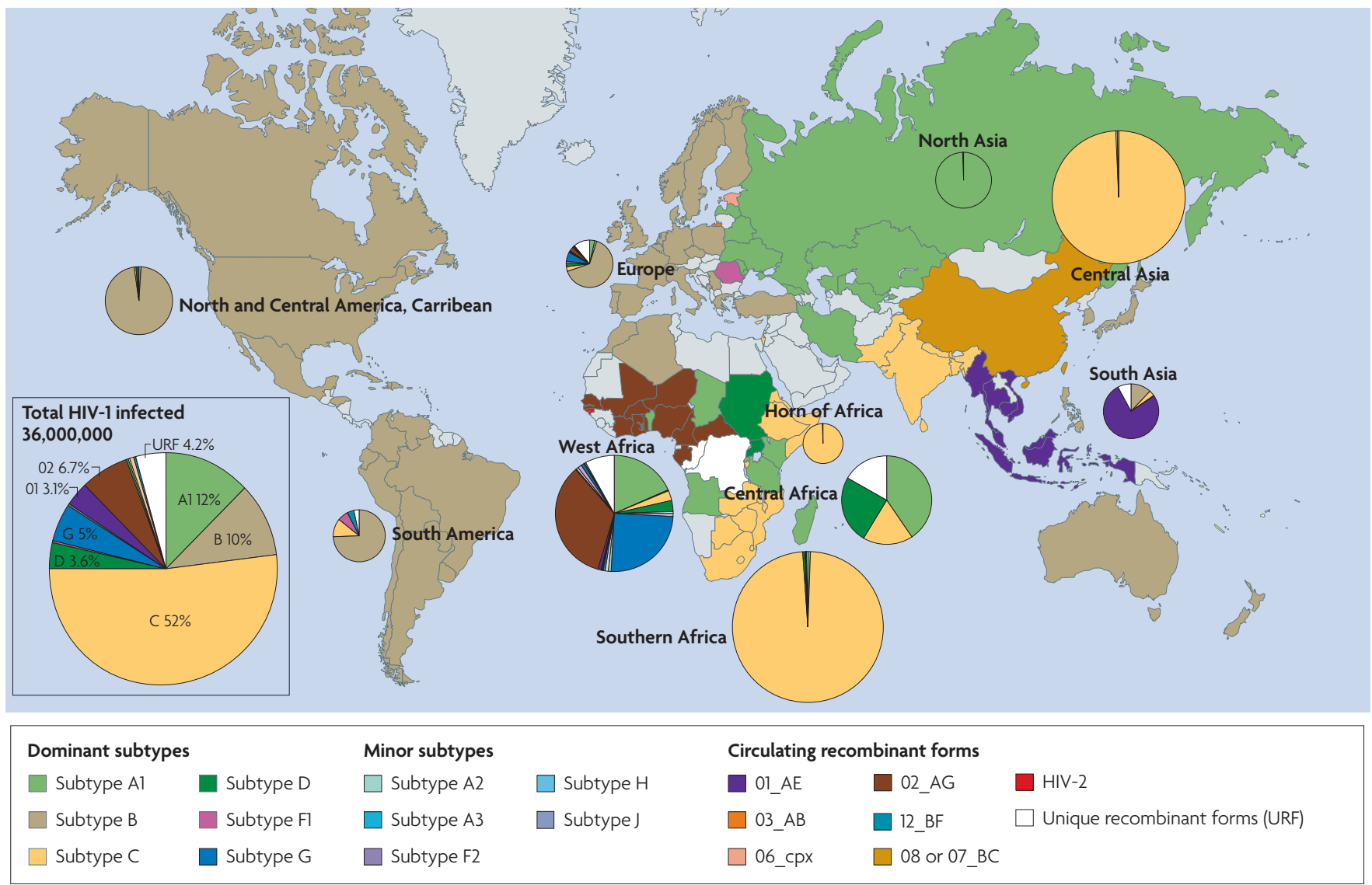

Figure 3 | HIV-1 diversity in the worldwide epidemic. The frequency of each HIV-1 subtype and recombinant form was estimated in each country based on published findings. A complete breakdown of subtype prevalence per country and the countries present in each region are listed in the Supplementary information S1 (table). The countries are colour-coded based on the dominant HIV-1 group main
(M) subtype. The countries coloured grey have a low level of HIV-1 prevalence or were not represented in the scientific literature related to HIV-1 subtype prevalence. The pie charts depict the proportion of each subtype or recombinant form in each geographical region. The size of the pies is proportional to the number of HIV-1 infected individuals in that particular region. 
progression from 1992-1998. Interestingly, the period following 1992 also corresponds to increasing use of combination ARV treatment, which could only be indirectly related to this attenuation as none of the patients received ARVs during the study period. It is possible that drug resistance mutations might have appeared at a higher frequency during sub-optimal treatment from 1992-1998, that is, before the widespread use of highly active antiretroviral therapy (HAART). These mutations have been associated with reduced fitness ${ }^{95,96}$ but their presence following a new transmission and in the absence of ARV treatment is low $(<5-20 \%)^{122,123}$ and would not fully explain why the rates of disease progression might have decreased between 1992-1998.

The detection of fluctuations in the rate of $\mathrm{CD} 4^{+} \mathrm{T}$-cell decline at the start of the HIV-1 epidemic in Europe is surprising given the preliminary data suggesting that replicative fitness might have been higher in the 1980s compared with the 2000s (REF. 121). Slopes of $\mathrm{CD} 4^{+} \mathrm{T}$-cell decline can be heavily influenced by the length of longitudinal follow-up, the follow-up period post-infection, and the number of data points ${ }^{124}$. For example, most patients in the 1980s presented to a clinic with advanced disease when the slopes of CD4+ $4^{+}$-cell decline are commonly lower than in early disease (especially once the level falls below $200 \mathrm{CD} 4^{+} \mathrm{T}$ cells per $\mu \mathrm{l})^{124,125}$. Therefore, 'slow' disease progression during 1986-1988 might be associated with the accrual of patients with more advanced disease. In general, natural history cohorts starting at early or acute infection and ending with AIDS-defining illnesses or morbidity (typically 3-10 years without ARV treatment) provide the best estimates of $\mathrm{CD}^{+} \mathrm{T}$-cell decline and disease progression rates ${ }^{125}$. In the developed world, cohorts from the 1980s are not readily available owing to late diagnosis, whereas the current cohorts are difficult to assess for the natural history of disease due to ARV intervention. Finally, given the reduced HLA diversity and greater ethnic homogeneity in the Caucasian population, the attenuation rate of subtype $B$ HIV-1 virulence might be less dramatic than the attenuation possible with non-B subtypes in sub-Saharan Africa.

\section{In vivo and ex vivo fitness differences}

Early observations on the rates of disease progression in west Africa showed that HIV-2 infections were less aggressive than HIV-1 infections ${ }^{126,127}$. HIV-2 is also transmitted less frequently than HIV-1 in human populations of similar demographics and similar opportunities for sexual contacts ${ }^{128,129}$. Whereas HIV-1 has spread across the globe, founder events of HIV-2 in areas other than west Africa did not result in regional epidemics (FIG. 3). Even HIV-1 infections are not evenly distributed among the different groups and subtypes: group $\mathrm{N}$ infections have only been characterized in five Cameroonians, whereas $\sim 30,000$ group $\mathrm{O}$ infections are estimated in Cameroon and Gabon ${ }^{6,46}$.

HIV-1 group $M$ subtype $C$ has spread more rapidly than any other $\mathrm{M}$ subtype, partly as a result of recent subtype $\mathrm{C}$ pandemics in southern Africa, south America and Asia ${ }^{130-133}$ (FICS 3,4a; Supplementary information S1 (table)). 'Pure' HIV-1 subtype C or HIV-1 recombinant forms containing at least the envelope gene of subtype $\mathrm{C}$ are now responsible for $>50 \%$ of the $\mathrm{HIV}-1$ infections worldwide ${ }^{130}$. A founder event of subtype C or a C-containing recombinant form in many regions has also resulted in an apparent displacement of existing HIV-1 subtypes (such as subtypes B and CRF01_AE in south China ${ }^{131,132}$, many subtypes in Kinshasa,

Democratic Republic of Congo ${ }^{134}$ and subtype B in southern Brazil ${ }^{133}$ ) (FICS 3,4a). This displacement could be due to the introduction of subtype $\mathrm{C}$ into a population by different transmission routes (heterosexual transmission versus intravenous drug use) or increased sexual activity (for example, commercial sex workers) ${ }^{135}$. A subtype might initially predominate in a specific transmission group but there is little supporting evidence to suggest that subtypes A, B, C, D and CRF01_AE (also referred to as $\mathrm{E}$ ) are any more or less transmissible by a specific route, in a specific ethnic group or in specific cell types ${ }^{53,136-141}$. In addition, such initial predominance is generally lost during an epidemic. For example, in Thailand HIV-1 subtypes B and CRF01_AE were segregated to intravenous drug users and heterosexuals, respectively, in the late 1980s but are now more uniformly mixed in the Thai population ${ }^{142}$.

Recent studies have attempted to establish the ex vivo 'pathogenic' and 'transmission' fitness of different HIV types, groups and subtypes ${ }^{52}$. Ex vivo 'pathogenic fitness' has been previously described as the relative replication capacity of HIV-1 in primary blood cells (CD4 ${ }^{+} \mathrm{T}$ cells and PBMCs), which in turn, might be related to HIV-1 virulence ${ }^{30,53}$. By contrast, ex vivo 'transmission fitness' is measured by competing primary HIV-1 isolates in cells of the dendritic lineage, which are derived from blood monocytes or human skin ${ }^{53,143}$. Future dual HIV-1 competition experiments using vaginal or cervical biopsy explants might be the best model for transmission fitness ${ }^{65,66}$.
To first establish the 'pathogenic fitness' (or virulence) of HIV, pair-wise competitions were done with eight HIV-2, six HIV-1 group $\mathrm{O}$ and $15 \mathrm{HIV}-1$ group $\mathrm{M}$ isolates (2 subtype A, 5 subtype B, 4 subtype C, 2 subtype D and 2 subtype CRF01_AE) in $\mathrm{PBMCs}^{144}$ (FIG. 4b). These viruses were classified as using either the CCR5 (R5) or the CXCR4 (X4) co-receptor for entry. When HIV isolates of the same phenotype (X4 or R5) were competed against each other, the following fitness order (based on $>2,000$ competitions) was observed (FIG. 4b): HIV-1 group M (subtype A, B, D and CRF01_AE) had a greater fitness than subtype $C$, which in turn was greater than HIV-2, and HIV-1 group $\mathrm{O}$ showed the lowest fitness.

With the exception of subtype C, this order reflects the prevalence of these human lentiviruses in the human population and the proposed rates of transmission efficiency $^{128,129}$. The R5 HIV-1 subtype C isolates were at least 100-fold less fit than HIV-1 isolates of any other group M subtype $\mathrm{e}^{53}$. Aside from subtype C, no other HIV-1 subtype has shown a significant fitness difference in group $\mathrm{M}^{53,144}$. Subtype C HIV-1 isolates are preferentially CCR5-tropic and non-syncytium-inducing (NSI) throughout disease, whereas infections with HIV-1 isolates of other subtypes result in a switch from CCR5 to CXCR4-tropic virus in approximately $50 \%$ of late disease cases. A few rare $\mathrm{X} 4$ subtype $\mathrm{C}$ isolates have been obtained from infected Zimbabweans late in disease ${ }^{145}$ but even the switch from R5 to X4 did not restore fitness, compared with other group $\mathrm{M}$ isolates (Nankya, I. et al, unpublished data).

\section{What causes differential spread?}

Differential spread of human lentiviruses in the host population is controlled by both transmission efficiency and opportunity, which is collectively referred to as $\mathrm{R}_{0}$ (REF. 22). Several models of microparasite expansion in humans have been proposed ${ }^{20,22,33,146,147}$ but most assume that during the early stages of the epidemic, the rate of infection $(d Y / d t)$ simply increases as a function of the number of infected individuals $(Y)$ during an infectious period ( $v=$ rate of moving into a noninfectious state), the probability of infecting a new host $(\beta)$ and the frequency of sexual contact (c) (REF. 22).

$$
d Y / d t \sim(\beta c-v) Y
$$

The opportunities for HIV-1 transmission relate to the number of sexual contacts, which are more frequent during acute infection and in the asymptomatic period of disease than during AIDS. An infected individual (without 
a
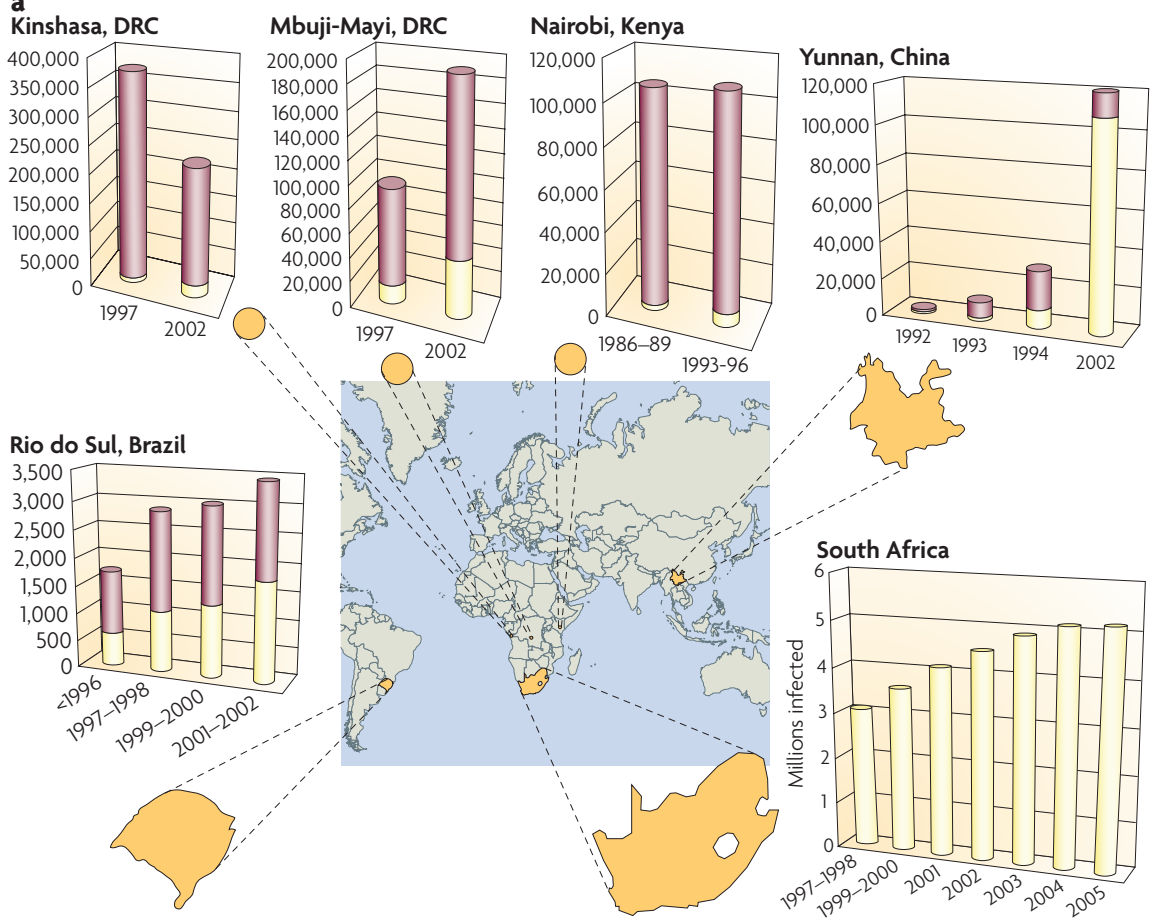

b
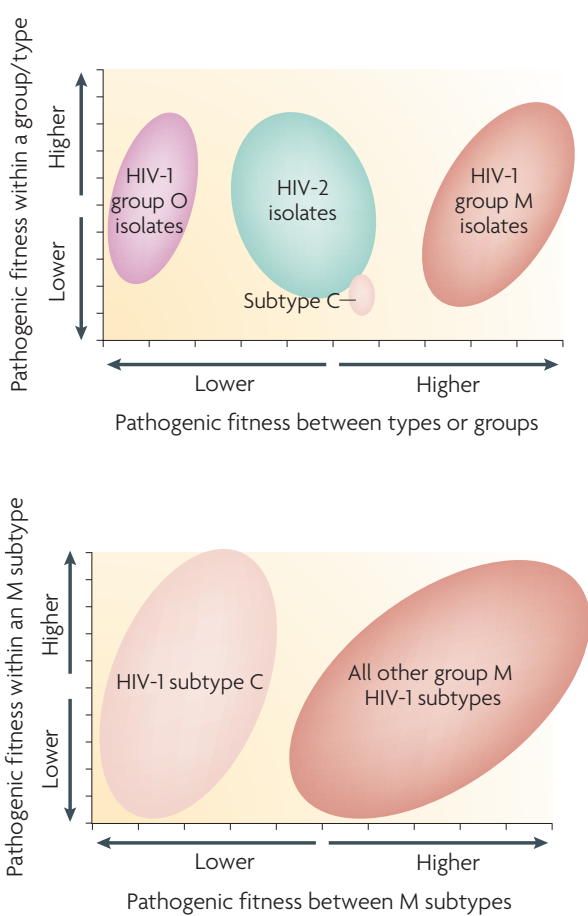

Figure 4 | Relationship between the increasing prevalence of HIV-1 subtype $C$ and its low pathogenic fitness. The prevalence of HIV-1 subtype $\mathrm{C}$ or subtype $\mathrm{C}$-containing recombinant forms has increased in proportion to other HIV-1 subtypes in Rio do Sul, Brazil (from 35\% in 1996 to $52 \% 2002)^{133}$, in Kinshasa and Mbuji-Mayi, Democratic Republic of Congo (DRC) (from $2.1 \%$ and $16.3 \%$ in 1997 to $9.7 \%$ and $25 \%$ in 2002, respectively) ${ }^{134}$ and in Yunnan, China (from 5.1\% in 1992 to $90 \%$ in 2002) ${ }^{162,163}$. By contrast, subtype $\mathrm{C}$ is dominant in South Africa ${ }^{164}$ and did not increase in proportion to subtypes $A$ and $D$ or recombinant forms in Kenya ${ }^{165}$. The number of HIV-1 cases increased in all of these regional epidemics (except in Kinshasa) ${ }^{6}$. a | The number of HIV-1 infections in specific years is subdivided into subtype C (yellow) and non-subtype-C (maroon) HIV-1 infections. b | A schematic representation of the pathogenic fitness of human lentiviruses, derived from $>3,000$ pair-wise dual HIV-1 competition experiments $^{26,27,49,50,108,120,123,125,165}$. The coloured ovals plot the fitness of primary HIV-1 isolates competed against isolates of the same type, group or subtype (y axis; mean fitness of 1 or equal fitness) compared with the fitness of primary HIV-1 isolates competed against isolates of different types, groups or subtypes. Each oval encompasses the fitness ( $\mathrm{x}$ and $\mathrm{y}$ fitness values) of at least 10 to 20 primary HIV-1 isolates. M, main; O, other. general knowledge of the transmission routes or infection status) could engage in several sexual encounters during the acute or early phase (approximately 3 months) ${ }^{148}$, the period of highest HIV-1 load ${ }^{61,62}$. Therefore, the exponential growth of HIV-1 infections in the early 1980 s could have been primarily the result of transmission from acutely infected men having sex with men or from intravenous drug users ${ }^{148-150}$ (BOX 1). However, in heterosexual populations with a reduced frequency of sexual contacts, a donor might transmit HIV-1 to more recipients during a long asymptomatic period ( $2-5$ years) of infection, with moderate viral load, than during the acute or early infection stage (1-3 months), with high viral load. Low HIV-1 virulence, which could lead to longer asymptomatic periods, could also increase the opportunity for transmission (BOX 1).

Similarly, poor fitness of HIV-1 group O and HIV-2 compared with HIV-1 group $M$ isolates was found when competitions were carried out in both PBMCs (ex vivo pathogenic fitness) and in dendritic-T-cell cultures (ex vivo transmission fitness) $)^{144}$. In relation to this ex vivo model, poor transmission efficiency has been well documented for HIV-2 compared with HIV-1 in the human populations in which both circulate $^{128,129}$. By contrast, subtype C HIV-1 isolates had similar ex vivo transmission fitness $^{53}$ compared with group M subtype B isolates in Langerhans cells even though subtype $\mathrm{C}$ isolates are at least 100 -fold less fit in PBMC cultures $^{53}$. In vivo observations indicate that subtype C HIV-1 is transmitted as efficiently as other HIV-1 group M isolates in human cohorts ${ }^{135,137}$ and in rhesus macaques, through the use of SIV-HIV env chimeric virus (SHIV-MJ4) ${ }^{151}$. These ex vivo and in vivo observations imply that HIV-1 subtype $\mathrm{C}$ is efficiently transmitted but is less virulent than other HIV-1 group $M$ isolates.

HIV-2 and HIV-1 groups $\mathrm{O}$ and $\mathrm{N}$ might have had limited expansion in the human population due to poor host adaptation and transmission efficiencies. By contrast, HIV-1 group $\mathrm{M}$ seems to be more virulent as well as more transmissible. Therefore, the progenitor of the HIV-1 group M lineage might have been more 'fit' for human infection, promoting further adaptation through rapid evolution and passage through the human population. New founder events in segregated human populations in central Africa might have led to subsequent divergence into subtypes. HIV-1 subtypes probably evolved and diverged under similar selection pressures, unless infecting a human population with distinct genetic polymorphisms that had an effect on virulence. Adaptation to the human population followed by attenuation of lethal virulence might, however, be occurring at different rates for divergent HIV-1 clades. If diversity in HLA types has a significant role in HIV-1 evolution, subtypes predominating in Africa, such as subtype C, might attenuate virulence at a faster rate than HIV-1 subtype B, which infects the Caucasian population of lower HLA diversity in the developed world. 


\section{Prevalence and reduced virulence}

HIV-1 subtype $\mathrm{C}$ might be in a more advanced stage of attenuation than other HIV-1 subtypes ${ }^{53,96,144}$. If it is less virulent than other subtypes, subtype $\mathrm{C}$ infections might result in slower disease progression, longer periods of asymptomatic infection and more opportunities for transmission; that is, it would be associated with an increase in $\mathrm{R}_{0}$ (see equation [1]; BOX 1). To support a model of disproportionate expansion of HIV-1 subtype $C$ in the human population (FIGS 3,4a), the transmission efficiency of subtype $\mathrm{C}$ must be greater than, or comparable to, other HIV-1 group M strains. Preliminary data suggest that subtype $\mathrm{C}$ is transmitted as efficiently as other HIV-1 group M subtypes $^{53,135,137,151}$. Therefore, decreased virulence could have a key role in the spread of subtype $\mathrm{C}$ by increasing the opportunity for transmission ${ }^{33}$. So far, comparisons of cohorts infected with subtype $\mathrm{C}$ and other subtypes show similar or higher viral loads in the subtype C-infected individuals ${ }^{152,153}$. These initial observations would argue against the possible subtype $\mathrm{C}$ attenuation and would support the more popular assumption, that subtype $\mathrm{C}$ is more virulent.

However, there are few studies on the natural history and progression of patients infected by any HIV-1 subtype other than subtype $\mathrm{B}^{154-157}$. Two studies have described faster disease progression in individuals infected with subtype D compared with subtype A- or subtype C-infected individuals in Uganda and Tanzania ${ }^{154-156}$. The possibility of greater subtype D virulence has been attributed to a higher propensity to switch from the NSI/R5 phenotype to the more aggressive syncytium-inducing (SI)/X4 phenotype ${ }^{158}$. By contrast, subtype $\mathrm{C}$ rarely switches from the NSI/R5 to the SI/X4 phenotype. At a recent presentation at the sixteenth International AIDS Conference in Toronto, Canada ${ }^{159}$, interim analyses of disease progression were described in a cohort of 256 subtype A-, C- and D-infected women, recruited at acute or early infection and followed for a mean of 36 months. The mean slope of $\mathrm{CD} 4^{+} \mathrm{T}$-cell decline in the subtype C-infected women from Zimbabwe was 2.3-fold slower than in subtype A- and D-infected women from Uganda $(p<0.008)$. However, there were no differences in the mean viral loads at 3 and 12 months between the countries or between different subtypes. Slower disease progression, as described by slower declines in $\mathrm{CD} 4^{+} \mathrm{T}$-cell counts, was not attributable to any other factors (such as opportunistic infections, sexual activity, diet, age and weight) apart from being infected with subtype $\mathrm{C}$ versus subtype A or D. In the Bantu corridor extending from Zimbabwe to Uganda, the frequency of HLA-A, -B and -C alleles and other host polymorphisms (for example, CCR5 deletions or promoter polymorphisms) are similar and could rule out obvious human genetic differences skewing HIV-1 evolution ${ }^{108}$.

\section{Summary}

The attenuation of infectious pathogens remains contentious and many doubt that the spread of HIV-1 through the human population will lead to decreased virulence as HIV-1 isolates actually gain replicative (or pathogenic) fitness during infection of human hosts. However, this gain might not be sufficient to reverse the loss in pathogenic fitness that occurs during transmission and early disease. The extreme genetic bottleneck following transmission reduces the HIV-1 population size and diversity by at least 10,000 -fold. In vitro studies indicate that this genetic reduction correlates with a significant fitness loss. The few HIV-1 clones establishing infection must evade the immune system, resulting in yet another series of genetic bottlenecks reducing fitness. Therefore, attenuation of virulence might occur if the net fitness loss during transmission or early disease is greater than the increase in pathogenic fitness during disease progression and between transmission events. Greater diversity in HLA alleles in African populations compared with Caucasian populations might result in stronger immune pressure in the human population and possibly faster rates of HIV-1 attenuation. Is the global dominance of HIV-1 subtype $C$ related to an attenuation of virulence? Does subtype $\mathrm{C}$ infection lead to slower disease progression and more opportunities for new transmission events? These questions might hold the key to the future of the HIV-1 epidemic and could provide a new focus for ARV treatment and vaccine design.

Kevin K. Ariën and Guido Vanham are at the HIV and Retrovirology Research Unit, Department of Microbiology, Institute of Tropical Medicine, Nationalestraat 155, B-2000 Antwerp, Belgium.

Kevin K. Ariën is currently at the Department of Clinical Chemistry, Microbiology and Immunology, Faculty of Medicine and Health Sciences, Ghent University, De Pintelaan 185, B-9000 Ghent, Belgium.

Eric J. Arts is at the Division of Infectious Diseases, Department of Medicine, Case Western Reserve University, 2109, Adelbert Rd, Cleveland, Ohio, 44195 USA

Correspondence to E.J.A e-mail:eja3@po.cwru.edu doi:10.1038/nrmicro1594 Published online 4 January 2007
Lemey, P., Pybus, O. G., Van, D. S. \& Vandamme, A. M A Bayesian statistical analysis of human T-cell lymphotropic virus evolutionary rates. Infect. Genet. Evol. 5, 291-298 (2005).

2. Van, D. S., Salemi, M. \& Vandamme, A. M. Dating the origin of the African human T-cell lymphotropic virus type-i (HTLV-I) subtypes. Mol. Biol. Evol. 18, 661-671 (2001).

3. Yoshida, M. Discovery of HTLV-1, the first human retrovirus, its unique regulatory mechanisms, and insights into pathogenesis. Oncogene $\mathbf{2 4}$, 5931-5937 (2005).

4. Coulthart, M. B., Posada, D., Crandall, K. A. \& Dekaban, G. A. On the phylogenetic placement of human T cell leukemia virus type 1 sequences associated with an Andean mummy. Infect. Genet. Evol. 6, 91-96 (2006).

5. Korber, B. et al. Timing the ancestor of the HIV-1 pandemic strains. Science 288, 1789-1796 (2000).

6. UNAIDS/WHO. AIDS Epidemic Update 2006. [online], http://www.unaids.org/en/HIV_data/epi2006/ (UNAIDS/WHO, Geneva, 2006).

7. Geisbert, T. W. \& Jahrling, P. B. Exotic emerging viral diseases: progress and challenges. Nature Med. 10 S110-S121 (2004).

8. Doherty, P. C., Turner, S. J., Webby, R. G. \& Thomas, P. G. Influenza and the challenge for immunology. Nature Immunol. 7, 449-455 (2006).

9. Perlman, S. \& Dandekar, A. A. Immunopathogenesis of coronavirus infections: implications for SARS. Nature Rev. Immunol. 5, 917-927 (2005).

10. Gage, K. L. \& Kosoy, M. Y. Natural history of plague: perspectives from more than a century of research. Annu. Rev. Entomol. 50, 505-528 (2005).

11. Drancourt, M., Houhamdi, L. \& Raoult, D. Yersinia pestis as a telluric, human ectoparasite-borne organism. Lancet Infect. Dis. 6, 234-241 (2006).

12. Fenner, F. \& Radcliffe, F. N. Myxomatosis (Cambridge University Press, Cambridge, 1965)

13. Zuniga, M. C. A pox on thee! Manipulation of the host immune system by myxoma virus and implications for viral-host co-adaptation. Virus Res. 88, 17-33 (2002)

14. Best, S. M. \& Kerr, P. J. Coevolution of host and virus: the pathogenesis of virulent and attenuated strains of myxoma virus in resistant and susceptible European rabbits. Virology 267, 36-48 (2000)

15. Knell, R. J. Syphilis in renaissance Europe: rapid evolution of an introduced sexually transmitted disease? Proc. Biol. Sci. 271, S174-S176 (2004).

16. Jin, M. J. et al. Mosaic genome structure of simian immunodeficiency virus from west African green monkeys. EMBO J. 13, 2935-2947 (1994)

17. Apetrei, C., Robertson, D. L. \& Marx, P. A. The history of SIVS and AIDS: epidemiology, phylogeny and biology of isolates from naturally SIV infected non-human primates (NHP) in Africa. Front. Biosci. 9, 225-254 (2004)

18. Courgnaud, V. et al. Identification of a new simian immunodeficiency virus lineage with a vpu gene present among different cercopithecus monkeys (C. mona, C. cephus, and C. nictitans) from Cameroon J. Virol. 77, 12523-12534 (2003).

19. Hassel, M. P. The Spatial and Temporal Dynamics of Host-Parasitoid Interactions (Oxford University Press, Oxford, 2000)

20. Diekmann, O. \& Hessterbeek, O. Mathematical Epidemiology of Infectious Diseases: Model Building. Analysis, and Interpretation (Wiley, Chichester, 2006).

21. Dronamraju, K. R. Infectious Diseases and HostPathogen Evolution (Cambridge University Press, Cambridge, 2004).

22. Anderson, R. M. \& May, R. M. Infectious Diseases of Humans: Dynamics and Control (Oxford University Press, Oxford, 1991).

23. Arts, E. J. \& Quinones-Mateu, M. E. Sorting out the complexities of HIV-1 fitness. AIDS 17, 780-781 (2003).

24. Quinones-Mateu, M. E. \& Arts, E. J. HIV-1 Fitness: Implications for Drug Resistance, Disease Progression, and Global Epidemic Evolution. [online], http://hiv.lanl.gov/content/hiv-db/

COMPENDIUM/2001/partI/Quinones.pdf (HIV Sequence Compendium, Theoretical Biology and Biophysics Group, Los Alamos National Laboratory, Los Alamos, 2001).

25. Feng, J. Y. $\&$ Anderson, K. S. Mechanistic studies examining the efficiency and fidelity of DNA synthesis by the 3TC-resistant mutant (184V) of HIV-1 reverse transcriptase. Biochemistry 38, 9440-9448 (1999).

26. Deval, J. et al. Mechanistic basis for reduced viral and enzymatic fitness of HIV-1 reverse transcriptase containing both K65R and M184V mutations. J. Biol. Chem. 279, 509-516 (2004). 
27. Bull, J. J. Virulence. Evolution 48, 1423-1437 (1994)

28. Bremermann, H. J. \& Pickering, J. A game-theoretical model of parasite virulence. J. Theor. Biol. 100, 411-426 (1983)

29. Troyer, R. M. et al. Changes in human immunodeficiency virus type 1 fitness and genetic diversity during disease progression. J. Virol. 79, 9006-9018 (2005).

30. Quinones-Mateu, M. E. et al. A dual infection/ competition assay shows a correlation between ex vivo human immunodeficiency virus type 1 fitness and disease progression. J. Virol. 74, 9222-9233 (2000).

31. Blaak, H., Brouwer, M., Ran, L. J., de Wolf, F. \& Schuitemaker, H. In vitro replication kinetics of human immunodeficiency virus type 1 (HIV-1) variants in relation to virus load in long-term survivors of HIV- 1 infection. J. Infect. Dis. 177, 600-610 (1998).

32. Barbour, J. D. et al. Higher CD4+ $\mathrm{T}$ cell counts associated with low viral pol replication capacity among treatment-naive adults in early HIV-1 infection. J. Infect. Dis. 190, 251-256 (2004).

33. Ewald, P. W. Evolution of Infectious Disease (Oxford University Press, Oxford, 1994).

34. Nowak, M. A. \& May, R. M. Virus Dynamics: Mathematical Principles of Immunology and Virology (Oxford University Press, Oxford, 2000).

35. Maynard, S. J. Group selection and kin selection. Nature 201, 1145-1147 (1964)

36. Szathmary, E. \& Maynard, S. J. From replicators to reproducers: the first major transitions leading to life. J. Theor. Biol. 187, 555-571 (1997).

37. Keele, B. F. et al. Chimpanzee reservoirs of pandemic and nonpandemic HIV-1. Science 313, 523-526 (2006).

38. Gao, F. et al. Origin of HIV-1 in the chimpanzee Pan troglodytes troglodytes. Nature 397, 436-441 (1999).

39. Santiago, M. L. et al. SIVcpz in wild chimpanzees Science 295, 465 (2002)

40. Van Heuverswyn, F. et al. Human immunodeficiency viruses: SIV infection in wild gorillas. Nature 444, 164 (2006).

41. Horimoto, T. \& Kawaoka, Y. Influenza: lessons from past pandemics, warnings from current incidents. Nature Rev. Microbiol. 3, 591-600 (2005).

42. Hirsch, V. M., Olmsted, R. A., Murphey-Corb, M., Purcell, R. H. \& Johnson, P. R. An African primate lentivirus (SIVsm) closely related to HIV-2. Nature 339, 389-392 (1989).

43. Lemey, P. et al. Tracing the origin and history of the HIV-2 epidemic. Proc. Natl Acad. Sci. USA 100 6588-6592 (2003).

44. Schim van der Loeff, M. F. \& Aaby, P. Towards a better understanding of the epidemiology of HIV-2. AIDS 13 S69-S84 (1999).

45. Schim van der Loeff, M. F. et al. Sixteen years of HIV surveillance in a West African research clinic reveals divergent epidemic trends of HIV-1 and HIV-2. Int J. Epidemiol. 35, 1322-1328 (2006).

46. Ayouba, A. et al. HIV-1 group O infection in Cameroon, 1986 to 1998. Emerg. Infect. Dis. 7, 466-467 (2001)

47. Yamaguchi, J. et al. HIV infections in northwestern Cameroon: identification of HIV type 1 group $O$ and dual HIV type 1 group $\mathrm{M}$ and group $\mathrm{O}$ infections. AIDS Res. Hum. Retroviruses 20, 944-957 (2004).

48. Yamaguchi, J. et al. HIV-1 Group N: evidence of ongoing transmission in Cameroon. AIDS Res. Hum Retroviruses 22, 453-457 (2006).

49. Yamaguchi, J. et al. Identification of HIV type 1 group $\mathrm{N}$ infections in a husband and wife in Cameroon: vira genome sequences provide evidence for horizontal transmission. AIDS Res. Hum. Retroviruses 22 83-92 (2006).

50. Salemi, M. et al. Dating the common ancestor of SIVcpz and HIV- 1 group $M$ and the origin of HIV- 1 subtypes using a new method to uncover clock-like molecular evolution. FASEB J. 15, 276-278 (2001).

51. Travers, S. A. et al. Timing and reconstruction of the most recent common ancestor of the subtype $C$ clade of human immunodeficiency virus type 1. J. Virol. 78 10501-10506 (2004).

52. Arien, K. K. et al. The replicative fitness of primary human immunodeficiency virus type 1 (HIV-1) group $\mathrm{M}$, HIV-1 group O, and HIV-2 isolates. J. Virol. 79, 8979-8990 (2005).

53. Ball, S. C. et al. Comparing the ex vivo fitness of CCR5-tropic human immunodeficiency virus type 1 isolates of subtypes B and C. J. Virol. 77, 1021-1038 (2003).
54. Barnett, S. W., Quiroga, M., Werner, A., Dina, D. \& Levy, J. A. Distinguishing features of an infectious molecular clone of the highly divergent and noncytopathic human immunodeficiency virus type 2 UC1 strain. J. Virol. 67, 1006-1014 (1993).

55. Talbott, R., Kraus, G., Looney, D. \& Wong-Staal, F. Mapping the determinants of human immunodeficiency virus 2 for infectivity, replication efficiency, and cytopathicity. Proc. Natl Acad. Sci. USA 90, 4226-4230 (1993)

56. Pollakis, G. et al. Phenotypic and genotypic comparisons of CCR5- and CXCR4-tropic human immunodeficiency virus type 1 biological clones isolated from subtype C-infected individuals. J. Virol. 78, 2841-2852 (2004).

57. Curran, J. W. et al. Epidemiology of HIV infection and AIDS in the United States. Science 239, 610-616 (1988).

58. Parazzini, F. et al. Number of sexual partners, condom use and risk of human immunodeficiency virus infection. Int J. Epidemiol, 24, 1197-1203 (1995).

59. Ryder, R. W. \& Behets, F. Reasons for the wide variation in reported rates of mother-to-child transmission of HIV-1. AIDS 8, 1495-1497 (1994).

60. Gray, R. H. et al. Probability of HIV-1 transmission per coital act in monogamous, heterosexual, HIV-1 discordant couples in Rakai, Uganda. Lancet 357 1149-1153 (2001).

61. Quinn, T. C. et al. Viral load and heterosexual transmission of human immunodeficiency virus type 1 . Rakai Project Study Group. N. Engl. J Med. 342 921-929 (2000)

62. Garcia, P. M. et al. Maternal levels of plasma human immunodeficiency virus type 1 RNA and the risk of perinatal transmission. Women and Infants Transmission Study Group. N. Engl. J Med. 341, 394-402 (1999)

63. Shankarappa, R. et al. Consistent viral evolutionary changes associated with the progression of human immunodeficiency virus type 1 infection. J. Virol. 73 10489-10502 (1999).

64. Zhu, T. et al. Genotypic and phenotypic characterization of HIV-1 patients with primary infection. Science 261 1179-1181 (1993)

65. Shattock, R. J. \& Moore, J. P. Inhibiting sexual transmission of HIV-1 infection. Nature Rev. Microbiol. 1, 25-34 (2003).

66. Shattock, R. J., Griffin, G. E. \& Gorodeski, G. I. In vitro models of mucosal HIV transmission. Nature Med. 6 , 607-608 (2000).

67. Bergstrom, C. T., McElhany, P. \& Real, L. A Transmission bottlenecks as determinants of virulence in rapidly evolving pathogens. Proc. Natl Acad. Sci USA 96, 5095-5100 (1999).

68 Lazaro, E., Escarmis, C., Perez-Mercader, J., Manrubia, S. C. \& Domingo, E. Resistance of virus to extinction on bottleneck passages: study of a decaying and fluctuating pattern of fitness loss. Proc. Natl Acad. Sci. USA 100, 10830-10835 (2003).

69. Elena, S. F. et al. Evolution of fitness in experimental populations of vesicular stomatitis virus. Genetics 142, 673-679 (1996)

70. Sagar, M. et al. Infection with multiple human immunodeficiency virus type 1 variants is associated with faster disease progression. J. Virol. 77 , 12921-12926 (2003)

71. Pontesilli, O. et al. Longitudinal analysis of human immunodeficiency virus type 1 -specific cytotoxic $T$ lymphocyte responses: a predominant gag-specific response is associated with nonprogressive infection. J. Infect. Dis. 178, 1008-1018 (1998).

72. Dyer, W. B. et al. Strong human immunodeficiency virus (HIV)-specific cytotoxic T- lymphocyte activity in Sydney blood bank cohort patients infected with nef-defective HIV type 1. J. Virol. 73, 436-443 (1999).

73. Cao, Y., Qin, L., Zhang, L., Safrit, J. \& Ho, D. D Virologic and immunologic characterization of longterm survivors of human immunodeficiency virus type 1 infection. N. Eng. J. Med. 332, 201-208 (1995).

74. Pantaleo, G. et al. Studies in subjects with long-term nonprogressive human immunodeficiency virus infection. N. Eng. J. Med. 332, 209-216 (1995).

75. Montefiori, D. C. et al. Neutralizing and infectionenhancing antibody responses to human immunodeficiency virus type 1 in long-term nonprogressors. J. Infect. Dis. 173, 60-67 (1996).

76. Carotenuto, P., Looij, D., Keldermans, L., de Wolf, F. \& Goudsmit, J. Neutralizing antibodies are positively associated with $\mathrm{CD} 4^{+} \mathrm{T}$-cell counts and T-cell function in long-term AIDS-free infection. AIDS 12 1591-1600 (1998)
77. Rosenberg, E. S. et al. Vigorous HIV-1-specific CD4 $\mathrm{T}$ cell responses associated with control of viremia. Science 278, 1447-1450 (1997).

78. Dean, M. et al. Genetic restriction of HIV-1 infection and progression to AIDS by a deletion allele of the CKR5 structural gene. Science 273, 1856-1862 (1996).

79. Berger, E. A., Murphy, P. M. \& Farber, J. M Chemokine receptors as HIV-1 coreceptors: roles in viral entry, tropism, and disease. Annu. Rev. Immunol. 17, 657-700 (1999).

80. Gonzalez, E. et al. Global survey of genetic variation in CCR5, RANTES, and MIP- $1 \alpha$ : impact on the epidemiology of the HIV-1 pandemic. Proc. Natl Acad. Sci. USA 98, 5199-5204 (2001).

81. Gonzalez, E. et al. The influence of CCL3L1 genecontaining segmental duplications on HIV-1/AIDS susceptibility. Science 307, 1434-1440 (2005).

82. McDermott, D. H. et al. CCR5 promoter polymorphism and HIV-1 disease progression. Multicenter AIDS Cohort Study (MACS). Lancet 352. 866-870 (1998)

83. Mellors, J. W. et al. Prognosis in HIV-1 infection predicted by the quantity of virus in plasma. Science 272, 1167-1170 (1996).

84. Collier, A. C. et al. Treatment of human immunodeficiency virus infection with saquinavir, zidovudine, and zalcitabine. AIDS Clinical Trials Group N. Engl. J. Med. 334, 1011-1017 (1996).

85. Silvestri, G. et al. Nonpathogenic SIV infection of sooty mangabeys is characterized by limited bystander immunopathology despite chronic high-level viremia. Immunity. 18, 441-452 (2003).

86. Rodriguez, B. et al. Predictive value of plasma HIV RNA level on rate of CD4 T-cell decline in untreated HIV infection. JAMA 296, 1498-1506 (2006).

87. Deacon, N. J. et al. Genomic structure of an attenuated quasispecies of HIV-1 from a blood transfusion donor and recipients. Science $\mathbf{2 7 0}$ 988-991 (1995).

88. Kirchhoff, F., Greenough, T. C., Brettler, D. B., Sullivan, J. L. \& Desrosiers, R. C. Brief report: absence of intact nef sequences in a long-term survivor with nonprogressive HIV-1 infection. N. Eng. J. Med. 332 228-232 (1995)

89. Huang, Y., Zhang, I. \& Ho, D. D. Biological characterization of nef in long-term survivors of human immunodeficiency virus type 1 infection. J. Virol. 69 8142-8146 (1995).

90. Leroux, C., Issel, C. J. \& Montelaro, R. C. Novel and dynamic evolution of equine infectious anemia virus genomic quasispecies associated with sequential disease cycles in an experimentally infected pony. J. Virol. 71, 9627-9639 (1997)

91. Plagemann, P. G., Chen, Z. \& Li, K. Replication competition between lactate dehydrogenase-elevating virus quasispecies in mice. Implications for quasispecies selection and evolution. Arch. Virol. 146 1283-1296 (2001).

92. Lu, J. \& Kuritzkes, D. R. A novel recombinant marker virus assay for comparing the relative fitness of hiv-1 reverse transcriptase variants. J. Acquir. Immune. Defic. Syndr. 27, 7-13 (2001).

93. Holland, J. J., de la Torre, J. C. Clarke, D. K. \& Duarte, E. Quantitation of relative fitness and great adaptability of clonal populations of RNA viruses. J. Virol. 65, 2960-2967 (1991).

94. Domingo, E., Escarmis, C., Menendez-Arias, L. $\delta$ Holland, J. in Origin And Evolution of Viruses (eds Domingo, E., Webster, R. \& Holland, J.) 141-16 (Academic Press, San Diego, 1999).

95. Harrigan, P. R., Bloor, S. \& Larder, B. A. Relative replicative fitness of zidovudine-resistant human immunodeficiency virus type 1 isolates in vitro. J. Virol. 72, 3773-3778 (1998)

96. Quinones-Mateu, M. E. \& Arts, E. J. Virus fitness: concept, quantification, and application to HIV population dynamics. Curr. Top. Microbiol. Immunol. 299, 83-140 (2006)

97. Clarke, D. K. et al. The red queen reigns in the kingdom of RNA viruses. Proc. Natl Acad. Sci. USA 91 4821-4824 (1994).

98. Novella, I. S. et al. Exponential increases of RNA virus fitness during large population transmissions. Proc. Natl Acad. Sci. USA 92, 5841-5844 (1995).

99. Chao, L. Fitness of RNA virus decreased by Muller's ratchet. Nature 348, 454-455 (1990).

100. Domingo, E. \& Holland, J. J. RNA virus mutations and fitness for survival. Annu. Rev. Microbiol. $\mathbf{5 1}$ 151-178 (1997) 
101. Yuste, E., Sanchez-Palomino, S., Casado, C. Domingo, E. \& Lopez-Galindez, C. Drastic fitness loss in human immunodeficiency virus type 1 upon serial bottleneck events. J. Virol. 73, 2745-2751 (1999).

102. Mullins, J. I. \& Jensen, M. A. Evolutionary dynamics of HIV-1 and the control of AIDS. Curr. Top. Microbiol. Immunol. 299, 171-192 (2006).

103. Wei, X. et al. Viral dynamics in human immunodeficiency virus type 1 infection. Nature 373 117-122 (1995)

104. Betts, M. R. et al. Analysis of total human immunodeficiency virus (HIV)-specific CD4 ${ }^{+}$and $C D 8^{+}$ T-cell responses: relationship to viral load in untreated HIV infection. J. Virol. 75, 11983-11991 (2001).

105. Rosenberg, E. S. et al. Immune control of HIV-1 after early treatment of acute infection. Nature 407, 523-526 (2000)

106. Goulder, P. J. et al. Substantial differences in specificity of HIV-specific cytotoxic T cells in acute and chronic HIV infection. J. Exp. Med. 193, 181-194 (2001).

107. Goulder, P. J. \& Watkins, D. I. HIV and SIV CTL escape: implications for vaccine design. Nature Rev. Immunol. 4, 630-640 (2004).

108. Cao, K. et al. Differentiation between African populations is evidenced by the diversity of alleles and haplotypes of HLA class I loci. Tissue Antigens 63, 293-325 (2004)

109. Moore, C. B. et al. Evidence of HIV-1 adaptation to HLA-restricted immune responses at a population level. Science 296, 1439-1443 (2002).

110. Goulder, P. J. et al. Evolution and transmission of stable CTL escape mutations in HIV infection. Nature 412, 334-338 (2001).

111. Leslie, A. J. et al. HIV evolution: CTL escape mutation and reversion after transmission. Nature Med. 10, 282-289 (2004)

112. Martinez-Picado, J. et al. Fitness cost of escape mutations in p24 Gag in association with control of human immunodeficiency virus type 1 . J. Virol. 80 3617-3623 (2006).

113. Troyer, R. M. et al. The fitness cost of CTL escape: not a terrible hardship on HIV-1? [online], http://www. iasociety.org/abstract/show.asp?abstract id = 2199593 (XVI International AIDS Conference, Toronto, Canada 13-18 August, 2006)

114. Sheehy, A. M., Gaddis, N. C., Choi, J. D. \& Malim, M. H. Isolation of a human gene that inhibits HIV-1 infection and is suppressed by the viral Vif protein. Nature 418, 646-650 (2002).

115. Stremlau, M. et al. The cytoplasmic body component TRIM5 $\alpha$ restricts HIV- 1 infection in Old World monkeys. Nature 427, 848-853 (2004).

116. Markowitz, M. et al. Infection with multidrug resistant, dual-tropic HIV-1 and rapid progression to AIDS: a case report. Lancet 365, 1031-1038 (2005).

117. Van de, P. P. Viral and host determinants of HIV-1 pathogenesis. AIDS 20, 933-934 (2006).

118. Muller, V. \& De Boer, R. J. The integration hypothesis: an evolutionary pathway to benign SIV infection. PLoS Pathog. 2, e 15 (2006).

119. Muller, V. et al. Stable virulence levels in the HIV epidemic of Switzerland over two decades. AIDS 20 , 889-894 (2006).

120. Kannangara, S., DeSimone, J. A. \& Pomerantz, R. J. Attenuation of HIV-1 infection by other microbial agents. J. Infect. Dis. 192, 1003-1009 (2005).

121. Arien, K. K. et al. Replicative fitness of historical and recent HIV-1 isolates suggests HIV- 1 attenuation over time. AIDS 19, 1555-1564 (2005)

122. Yerly, S. et al. Transmission of antiretroviral-drug resistant HIV-1 variants. Lancet 354, 729-733 (1999).

123. Turner, D. \& Wainberg, M. A. HIV transmission and primary drug resistance. AIDS Rev. 8, 17-23 (2006)

124. Eyster, M. E. Test may predict which patients with HIV infection will develop AIDS. Am. Fam. Physician 39, 276 (1989).

125. McCune, J. M. The dynamics of $C D 4^{+}$T-cell depletion in HIV disease. Nature 410, 974-979 (2001).

126. Marlink, R. et al. Reduced rate of disease development after HIV-2 infection as compared to HIV-1. Science 265, 1587-1590 (1994).

127. Matheron, S. et al. Factors associated with clinical progression in HIV-2 infected-patients: The French ANRS cohort. AIDS 17, 2593-2601 (2003).

128. Gilbert, P. B. et al. Comparison of HIV-1 and HIV-2 infectivity from a prospective cohort study in Senegal. Stat. Med. 22, 573-593 (2003).
129. Kanki, P. J. et al. Slower heterosexual spread of HIV-2 than HIV-1. Lancet 343, 943-946 (1994).

130. Essex, M. Human immunodeficiency viruses in the developing world. Adv. Virus Res. 53, 71-88 (1999).

131. Luo, C. C. et al. HIV-1 subtype C in China. Lancet 345 1051-1052 (1995)

132. Piyasirisilp, S. et al. A recent outbreak of human immunodeficiency virus type 1 infection in southern China was initiated by two highly homogeneous, geographically separated strains, circulating recombinant form $\mathrm{AE}$ and a novel $\mathrm{BC}$ recombinant. J. Virol. 74, 11286-11295 (2000).

133. Soares, E. A. et al. HIV-1 subtype C dissemination in southern Brazil. AIDS 19, S81-S86 (2005).

134. Vidal, N. et al. Distribution of HIV-1 variants in the Democratic Republic of Congo suggests increase of subtype C in Kinshasa between 1997 and 2002 J. Acquir. Immune. Defic. Syndr. 40, 456-462 (2005).

135. Walker, P. R., Pybus, O. G., Rambaut, A. \& Holmes, E. C. Comparative population dynamics of HIV-1 subtypes B and C: subtype-specific differences in patterns of epidemic growth. Infect. Genet. Evol. 5, 199-208 (2005).

136. Renjifo, B. et al. Differences in perinatal transmission among human immunodeficiency virus type 1 genotypes. J. Hum. Virol. 4, 16-25 (2001).

137. Renifo, B. et al. Preferential in utero transmission of HIV-1 subtype C as compared to HIV-1 subtype A or D. AIDS 18, 1629-1636 (2004)

138. Yang, C. et al. Genetic diversity of HIV-1 in western Kenya: subtype-specific differences in mother-to-child transmission. AIDS 17, 1667-1674 (2003).

139. Eshleman, S. H. et al. Comparison of mother-to-child transmission rates in Ugandan women with subtype A versus D HIV-1 who received single-dose nevirapine prophylaxis. HIV Network For Prevention Trials 012. J. Acquir. Immune. Defic. Syndr. 39, 593-597 (2005)

140. Pope, M. et al. Human immunodeficiency virus type 1 strains of subtypes B and $E$ replicate in cutaneous dendritic cell-T-cell mixtures without displaying subtype-specific tropism. J. Virol. 71, 8001-8007 (1997)

141. Dittmar, M. T. et al. Langerhans cell tropism of human immunodeficiency virus type 1 subtype A through $\mathrm{F}$ isolates derived from different transmission groups. J. Virol. 71, 8008-8013 (1997).

142. Tovanabutra, S et al. The changing molecular epidemiology of HIV type 1 among northern Thai drug users, 1999 to 2002. AIDS Res. Hum. Retroviruses 20, 465-475 (2004).

143. Zaitseva, M. et al. Expression and function of CCR5 and CXCR4 on human Langerhans cells and macrophages: implications for HIV primary infection. Nature Med. 3, 1369-1375 (1997)

144. Arien, K. K. et al. The replicative fitness of primary human immunodeficiency virus type 1 (HIV-1) group M, HIV-1 group O, and HIV-2 isolates. J. Virol. 79 , 8979-8990 (2005)

145. Johnston, E. R. et al. High frequency of syncytiuminducing and CXCR4-tropic viruses among human immunodeficiency virus type 1 subtype C-infected patients receiving antiretroviral treatment. J. Virol. 77, 7682-7688 (2003)

146. Gupta, S., Anderson, R. M. \& May, R. M. Networks of sexual contacts: implications for the pattern of spread of HIV. AIDS 3, 807-817 (1989).

147. Anderson, R. M., Ng, T. W., Boily, M. C. \& May, R. M The influence of different sexual-contact patterns between age classes on the predicted demographic impact of AIDS in developing countries. Ann. N. Y. Acad. Sci. 569, 240-274 (1989)

148. Pilcher, C. D., Eron, J. J. Jr, Galvin, S., Gay, C. \& Cohen, M. S. Acute HIV revisited: new opportunities for treatment and prevention. J. Clin. Invest. 113 937-945 (2004)

149. Jacquez, J. A., Koopman, J. S., Simon, C. P. \& Longini, I. M. Jr. Role of the primary infection in epidemics of HIV infection in gay cohorts. J. Acquir. Immune. Defic Syndr. 7, 1169-1184 (1994).

150. Koopman, J. S. et al. The role of early HIV infection in the spread of HIV through populations. J. Acquir. Immune. Defic. Syndr. Hum. Retrovirol. 14, 249-258 (1997).

151. Ndung'u, T. et al. Infectious simian/human immunodeficiency virus with human immunodeficiency virus type 1 subtype $C$ from an African isolate: rhesus macaque model. J. Virol. 75, 11417-11425 (2001).
152. Gray, C. M. et al. Viral dynamics and $C D 4^{+} \mathrm{T}$ cell counts in subtype $\mathrm{C}$ human immunodeficiency virus type 1-infected individuals from southern Africa. AIDS Res. Hum. Retroviruses 21, 285-291 (2005).

153. Neilson, J. R. et al. Subtypes of human immunodeficiency virus type 1 and disease stage among women in Nairobi, Kenya. J. Virol. 73 4393-4403 (1999).

154. Kaleebu, P. et al. Effect of human immunodeficiency virus (HIV) type 1 envelope subtypes A and D on disease progression in a large cohort of HIV-1-positive persons in Uganda. J. Infect. Dis. 185, 1244-1250 (2002).

155. Vasan, A. et al. Different rates of disease progression of HIV type 1 infection in Tanzania based on infecting subtype. Clin. Infect. Dis. 42, 843-852 (2006).

156. Kaleebu, P. et al. Relationship between HIV-1 Env subtypes $A$ and $D$ and disease progression in a rural Ugandan cohort. AIDS 15, 293-299 (2001).

157. Choge, I. et al. Genotypic and phenotypic characterization of viral isolates from HIV-1 subtype C-infected children with slow and rapid disease progression. AIDS Res. Hum. Retroviruses 22. 458-465 (2006).

158. Senkaali, D. et al. The relationship between HIV type 1 disease progression and V3 serotype in a rural Ugandan cohort. AIDS Res. Hum. Retroviruses 20, 932-937 (2004)

159. Arts, E. J. et al. Infection with subtype C HIV-1 of lowe replicative fitness as compared to subtypes $A$ and $D$ leads to slower disease progression in Zimbabwean and Ugandan women. [online], http://www.iasociety. org/abstract/show.asp?abstract_id $=2199440$ (XV) International AIDS Conference, Toronto, Canada 13-18 August, 2006)

160. Pierson, T., McArthur, J. \& Siliciano, R. F. Reservoirs for HIV-1: mechanisms for viral persistence in the presence of antiviral immune responses and antiretroviral therapy. Annu. Rev. Immunol. 18 665-708 (2000).

161. Finzi, D. et al. Identification of a reservoir for HIV-1 in patients on highly active antiretroviral therapy. Science 278, 1295-1300 (1997).

162. Li, D. Q., Zheng, X. W. \& Zhang, G. Y. Study on the distribution HIV-1 C subtype in Ruili and other counties, Yunnan, China (Translation). Zhonghua Liu Xing. Bing. Xue. Za Zhi. 17, 337-339 (1996).

163. Li, X. J. et al. Molecular epidemiology of the heterosexual HIV-1 transmission in Kunming, Yunnan Province of China suggests origin from the local IDU epidemic. AIDS Res. Hum. Retroviruses 21, 977-980 (2005).

164. Van Harmelen, J. H. et al A predominantly HIV type subtype C-restricted epidemic in South African urban populations. AIDS Res. Hum. Retroviruses 15, 395-398 (1999)

165. Rainwater, S. et al. No evidence for rapid subtype c spread within an epidemic in which multiple subtypes and intersubtype recombinants circulate. AIDS Res. Hum. Retroviruses 21, 1060-1065 (2005).

\section{Acknowledgements}

We thank Miguel Quinones-Mateu, Luc Kestens, Robert Colebunders and Guido van der Groen for their helpful suggestions in the development of these attenuation hypotheses. We also thank Aslam Syed and Lora Angelova in the Arts laboratory for their contributions to the supplementary data.

\section{Competing interests statement}

The authors declare no competing financial interests.

\section{DATABASES}

The following terms in this article are linked online to: Entrez Genome: http://www.ncbi.nlm.nih.gov/entrez/query. fcgi?db=genome HIV-1|HTLV|SIV

Entrez Genome Project: http://www.ncbi.nlm.nih.gov/ entrez/query.fcgi?db=genomeprj Yersinia pestis

FURTHER INFORMATION

Eric J. Art's homepage:

http://www.case.edu/med/microbio/artslab/

dbMHC web site:

http://www.ncbi.nlm.nih.gov/projects/mhc/ihwg.cgi

SUPPLEMENTARY INFORMATION

See online article: S1 (table)

Access to this links box is available online. 